\title{
CURRICULUM E INCLUSIÓN: el caso del curriculum para la Educación Primaria de Jóvenes y Adultos de la Provincia de Buenos Aires (Argentina)
}

Graciela Cappelletti

Universidad de Buenos Aires

Silvina Feeney

Universidad de General Sarmiento

\begin{abstract}
Resumen
El trabajo presenta el proceso social de diseño del curriculum para la Modalidad de Educación Primaria de Jóvenes y Adultos de una jurisdicción de la República Argentina. El trabajo se focaliza en tres decisiones de política curricular: la adopción de un enfoque participativo para la construcción del diseño o texto curricular resultante; la recuperación de un conjunto de normativa promulagada por el Consejo Federal de Educación (Argentina) en el marco de políticas educativas que impulsaron el derecho a la educación; la adopción de criterios curriculares que impulsan la integración de campos de conocimientos, la definición contextualizada del contenido y la flexibilización curricular como intento de atender la especificidad de los sujetos jóvenes y adultos.
\end{abstract}

Palabras clave: política curricular, educación de jóvenes y adultos, enfoque curricular participativo

\begin{abstract}
The work presents the social process of curriculum design for the Modality of Primary Education of Young People and Adults of a jurisdiction of the Argentine Republic. The work focuses on three curricular policy decisions: the adoption of a participatory approach to the construction of the resulting curriculum design or text; the recovery of a set of regulations promulgated by the Federal Council of Education (Argentina) within the framework of educational policies that promoted the right to education; the adoption of curricular criteria that promote the integration of fields of knowledge, contextualized definition of content and curricular flexibility as an attempt to address the specificity of young and adult subjects.
\end{abstract}

Keywords: curriculum policy, youth and adult education, participatory curriculum approach

\section{Resumo}

O trabalho apresenta o processo social de desenho curricular da Modalidade de Educação Primária de Jovens e Adultos de uma jurisdição da República Argentina. O trabalho se concentra em três decisões de política curricular: a adoção de uma abordagem participativa para a construção do design ou texto curricular resultante; a recuperação de um conjunto de regulamentos promulgados pelo Conselho Federal de Educação (Argentina) no âmbito de políticas educacionais que promovam o direito à educação; a adoção de critérios curriculares que promovam a integração de áreas do conhecimento, a definição contextualizada de conteúdo e a flexibilidade curricular como uma tentativa de abordar a especificidade de jovens e adultos.

Palavras-chave: política curricular, educação de jovens e adultos, abordagem participativa do currículo

ISSN 1645-1384 (online) www.curriculosemfronteiras.org 


\section{Introducción}

En este trabajo presentamos el proceso social de elaboración del Diseño Curricular para la escuela primaria de Jóvenes y Adultos de la Provincia de Buenos Aires (Argentina), que se dio entre los años 2015 a 2017. Entendemos que el diseño de un curriculum es un proceso social y culturalmente mediado. Así considerado, el curriculum además de un texto, alude a una serie de prácticas y de acciones que se desarrollan en distintos niveles y contextos institucionales. Un curriculum implica primordialmente un proceso de construcción social que se desarrolla en un marco impregnado de tradiciones de pensamiento pedagógico y curricular, frecuentemente diversas (Amantea et al, 2006).

En Argentina, en los primeros años del siglo XXI, en el marco de las denominadas "políticas de inclusión", se evidencia un reconocimiento de los derechos sociales y, en este sentido, se pone en la esfera pública el debate acerca de cómo garantizar el derecho a la educación vinculándolo al derecho a la vida, al trabajo, a la salud, etc. (Gentili, 2011; Arroyo, 2001). De allí la importancia que adquirieron distintos programas que procuraron atender los problemas de reducción del fracaso, mantenimiento de la escolaridad y mejora de los aprendizajes de alumnos de sectores desfavorecidos (Feeney y Feldman, 2016; Feldman, Atorresi, Mekler, 2013; Feldman, Amantea, Feced, 2014) y, las múltiples intervenciones destinadas a transformar las condiciones de trabajo docente y la organización del trabajo pedagógico.

A partir del año 2015, se asiste en Argentina a un cambio en las orientaciones de las políticas públicas como resultado de la llegada al poder de un gobierno pro mercado. El reciente escenario se distingue por importantes transformaciones económicas y culturales que rápidamente comenzaron a desplegarse, expresándose de manera particular en el campo de la educación. Aún así, el proceso de construcción curricular que estamos presentando, recupera los marcos legales promulgados en el período anterior, entre los años 2011 a 2013, y avanza en una fuerte reconceptualización del sujeto adulto en el marco de la preocupación por el derecho a la educación ${ }^{1}$.

En el marco de las preocupaciones de política educativa en tiempos predominantemente atravesados por estrategias neoliberales y conservadoras, la elaboración de una propuesta curricular que recupera las tradiciones de construcción colaborativa y de transformación social resulta un producto valioso para compartir, en tanto colabora con el debate teórico y las resoluciones prácticas que implica la realización de una propuesta curricular, en el caso que nos ocupa, destinada a la educación de Jóvenes y Adultos.

El trabajo se estructura en tres apartados que consideran los aspectos ligados al proceso social de diseño del curriculum desde un enfoque participativo, la recuperación de temas y problemas que señala la literatura acerca de procesos de reforma del curriculum en la región a partir de los desafíos de la inclusión educativa y, la presentación del caso del curriculum para la escuela primaria de Jóvenes y Adultos en la Provincia de Buenos Aires (Argentina). Por último, se identifican los aspectos pendientes de abordaje (y de diseño) en el marco de las políticas educativas que dieron lugar a la elaboración de esta propuesta. 


\section{Consideraciones para la adopción de un enfoque participativo para el diseño de un curriculum}

¿Cuándo cambia un diseño curricular? El modo tradicionalmente aceptado es que el curriculum cambia cuando una determinada gestión política se hace eco de "malestares" de la práctica del curriculum o, cuando la propia gestión política decide que son tiempos de “cambios” ya sea para revisar y actualizar contenidos, enfoques metodológicos, enfoques curriculares (las famosas competencias!) o una combinación de esos factores. En Argentina podemos destacar un par de procesos de renovación curricular en línea con estas preocupaciones.

Por un lado, la reforma educativa que se produce en el marco de la Ley Federal de Educación del año 1993, se funda en la preocupación sobre la falta de actualización del contenido en las escuelas argentinas (Frigerio, 1991). En esos años, desde la Facultad Latinoamericana de Ciencias Sociales (FLACSO), se sostiene la hipótesis de la "escuela vacía” y se abre una línea de reflexión sobre los contenidos de la enseñanza que luego se va a sostener desde el Ministerio de Cultura y Educación de la Nación con la edición de los Contenidos Básicos Comunes en el año 1995, impulsando una política de reforma del curriculum desde la renovación de los contenidos disciplinares. Por otro lado, el acuerdo alcanzado en el Consejo Federal de Cultura y Educación, entre el Ministerio Nacional, las provincias y la Ciudad de Buenos Aires, permitió establecer en el año 2004, Núcleos de Aprendizajes Prioritarios (NAP's), conformándose una base común para la enseñanza en todo el país. La fundamentación de su definición apela a la "Preocupación por la desigualdad y fragmentación del sistema”, que obliga a "identificar núcleos prioritarios que sirvan para cohesionar la práctica docente desde el nivel inicial hasta la educación polimodal/media"2. El texto de presentación de los NAP's va dirigido directamente a lo docentes acompañados de la promesa de una línea de trabajo que acompañará los NAP's mediante la elaboración de materiales de apoyo para la enseñanza, serie denominada "Cuadernos de aula” (Feeney, 2006).

Ahora bien, ¿cómo cambia un diseño curricular?, ¿cuáles son los procesos sociales que se habilitan para ello?. Por lo general, el cambio del curriculum se da desde la perspectiva “top-down”, caracterizada por procesos de mayor o menor participación de actores del sistema educativo -pero que en "casi” ningún caso implica al conjunto de quienes deberán implementar ese curriculum, los docentes-; el texto curricular resultante, llega a los docentes por mecanismos denominados top-down (Lundgren, 1996); es decir, desde el poder político a las escuelas.

¿Existe otra manera de generar cambios curriculares que no sea la establecida por la tradición?. Antes de analizar las respuestas teóricas que aporta la literatura sobre el curriculum, nos interesa destacar una experiencia llevada a cabo en el Asentamiento Universitario de San Martín de los Andes (Provincia del Neuquén, en la patagonia argentina), de la Universidad Nacional del Comahue (AUSMA), en el que desde 1975 funciona la carrera de Técnico Universitario en Forestación, a partir del trabajo participativo y fuertemente 
deliberativo de todos los claustros (Barco et al, 2005). En dicha experiencia, la demanda de un "técnico en educación" que se encargara del nuevo diseño, se revierte en una propuesta de trabajo desde la perspectiva de la investigación-acción del que se esperaba que emergiera -como así ocurrió- el nuevo diseño curricular, actualmente vigente.

La expectativa es, en general, que alguien "autorizado" indique qué y cómo hacer, instalando la demanda desde una perspectiva tecnicista, asumida por la figura del técnico o equipo técnico encargado. Desde una perspectiva radicalmente diferente, en esa experiencia citada, los "especialistas en curriculum" se ubicaron como investigadores, en la acción, acompañando el proceso de revisión del plan de estudios tanto como su reelaboración, aportando y sugiriendo cuando la tarea lo demandó y, ofreciendo los fundamentos de cada propuesta que se construyó en conjunto. También se revalorizaron saberes y experiencias de los docentes del AUSMA, de los graduados y estudiantes, así como rescatamos la propia historia de esta carrera, fuertemente ligada a la construcción territorial.

En síntesis, cada claustro, desde su ángulo, contribuyó a la constitución de una perspectiva rica y compleja. Entre todos se fue "escribiendo" el documento curricular, - que no es definitivo, sino abierto a instancias de cambio permanente- y cuyo inicio fue previo a cualquier "escritura", ya que tuvo lugar en las prácticas de docentes y alumnos, que precedieron a la modificación. Queda claro que se buscó romper con la tradición de pensar los contenidos de un plan desde la lógica de las asignaturas y los intereses de los docentes, entendidos como excluyentes de cualquier otro. Queda claro también, que la modificación se produce, entre otros aspectos, por la emergencia de una concepción diferente acerca del Técnico Forestal: aparece una propuesta que se opone a la hasta entonces vigente, que se ha ido generando desde el establecimiento de la carrera hasta el momento de su modificación (Goodson, 1995).

La experiencia citada introduce el debate otrora planteado desde la teoría curricular acerca de los enfoques teórico metodológicos adoptados para la elaboración del curriculum. Frente a enfoques que plantearon un procedimiento técnico, científicamente fundado para la elaboración del curriculum, que han constituido el modelo dominante en la teoría y práctica curricular y que se caracterizan por desarrollar una tecnología para aplicar a la construcción de programas y enfatizan la racionalización de los procedimientos (Tyler, 1949; Taba, 1962); se contraponen enfoques que plantearon un lenguaje práctico como forma de tratar el curriculum. Para Joseph Schwab (1969), principal defensor y representante del segundo enfoque, todos los problemas del campo del curriculum proceden de haber pretendido elaborar los curricula como deducción de los principios teóricos de unas u otras disciplinas, omitiendo que las cuestiones sobre qué y cómo enseñar surgen en las situaciones concretas. A su turno, es Lawrence Stenhouse $(1987,1991)$ quien comprende la práctica del curriculum como un proceso de investigación y propone reducir la distancia que media entre la intención y la realidad en el curriculum, lo que le da sentido a la teorización e investigación en el campo. Se proponen entender los curricula como procedimientos hipotéticos, regidos por unas ideas e intenciones educativas que los profesores podrían experimentar en clase. Para ambos, el método de construcción o elaboración del curriculum es la deliberación colegiada del cuerpo docente. 
Las teorías y tecnologías del curriculum han elaborado, a lo largo del siglo pasado, una importante batería de técnicas de diseño y evaluación curriculares que han colaborado para esta regulación y para definir la tarea docente como un trabajo básicamente técnico, cuyas reglas hay que seguir para alcanzar los productos proyectados desde las políticas. Sin embargo, desde que el curriculum tiene una inevitable dimensión práctica, en la acción de las escuelas, se puede afirmar que el mismo no puede ser dictaminado (ni controlado) "desde arriba”. También debe decirse que, en distintos contextos, han sido elaborados otros modos alternativos de entender y desarrollar el curriculum comprometidos con la producción de experiencias pedagógicas locales.

La adopción del enfoque participativo para la elaboración de la propuesta curricular del diseño de la escuela primaria de Jóvenes y Adultos de la Provincia de Buenos Aires

En principio, el diseño curricular a elaborar debía responder a las condiciones territoriales de implementación. En este sentido, presentamos los datos que expresan la cantidad de estudiantes y de centros educativos del nivel.

Cuadro: Unidades educativas, alumnos y secciones para el total de la provincia de Buenos Aires

\begin{tabular}{|c|c|c|c|c|c|c|c|c|c|}
\hline $\begin{array}{c}\text { Modalidad } \\
\text { Jóvenes y } \\
\text { Adultos }\end{array}$ & \multicolumn{3}{|c|}{ Total } & \multicolumn{3}{c|}{ Estatal } & \multicolumn{3}{c|}{ Privado } \\
\hline $\begin{array}{c}\text { Nivel } \\
\text { Primario }\end{array}$ & $\begin{array}{c}\text { Unidades } \\
\text { Educ. }\end{array}$ & Alumnos & Secciones & $\begin{array}{c}\text { Unidades } \\
\text { Educ. }\end{array}$ & Alumnos & Secciones & $\begin{array}{c}\text { Unidades } \\
\text { Educ. }\end{array}$ & Alumnos & Secciones \\
\cline { 2 - 11 } & 623 & 94.598 & 4.591 & 615 & 94.168 & 4.571 & 8 & 430 & 20 \\
\hline
\end{tabular}

Fuente: Dirección General de Escuelas de la Provincia de Buenos Aires. Relevamiento anual 2011.

Estos datos muestran la envergadura del caso analizado: ¿cuántos sujetos se forman? Pero también nos permite considerar las diversidades propias del nivel. Existen aulas de primaria de jóvenes y adultos en edificios escolares, también en edificios que "prestan" sus instalaciones para formar a jóvenes y adultos, y en cárceles. Los sujetos que asisten a escuelas de jóvenes y adultos tienen trayectorias escolares previas y edades diversas. Todo esto, además, en una provincia muy extensa en superficie y con configuraciones diversas que podríamos caracterizar en tres tipos principales: escuelas urbanas, escuelas rurales, escuelas del conurbano bonaerense.

Otro aspecto a considerar tiene que ver con la centralidad del sujeto adulto como destinatario de la formación. Hasta el momento, la propuesta curricular vigente devenía del área de nivel Primario. Es decir, el parámetro curricular era el diseño de escuela primaria, vigente para los niños con trayectorias escolares esperables para sus edades. Con lo cual, las decisiones de ese diseño estaban tomadas considerando como sujeto de la educación a los estudiantes de escolaridad básica. Proponer el diseño de un curriculum que se construya en torno a un sujeto adulto, constituyó una reparación para esa población que, como es posible 
anticipar, es una población vulnerada respecto a su derecho a la educación: quienes no cursaron la escolaridad primaria, y deciden hacerlo como adultos, efectivamente son sujetos que han tenido (y tienen) historias de vida complejas. Son sujetos que están atravesados por una historia de exclusión.

Este diseño curricular focaliza en los sujetos de la educación de jóvenes y adultos, con independencia de los otros niveles educativos, recuperando tradiciones y la historia de las especificidades de esta modalidad. Intenta marcar un cambio profundo y una continuidad en la concepción política-pedagógica de los sujetos destinatarios -siempre diversos, múltiples, portadores de prácticas, saberes, valores y hábitos diferentes entre sí-, proponiendo una estructura que pueda generar condiciones para la inclusión, la permanencia y la acreditación del nivel.

Esto nos llevó a considerar como principio para el diseño, además de lo mencionado, el reconocimiento de los adultos como sujetos portadores de saberes construidos por fuera de lo escolar, que les permite desenvolverse cotidianamente en la sociedad, en sus comunidades y en sus trabajos. Diseñar un curriculum que atienda estas especificidades resulta un desafío.

El proceso de elaboración curricular adoptó un enfoque participativo y contempló diferentes tareas. En primer lugar, análisis documental: de la normativa Federal, de la normativa Jurisdiccional -en tanto antecedentes que permiten considerar la viabilidad de la propuesta-, y finalmente, el análisis de propuestas de otras jurisdicciones de la Argentina, que nos permitieron considerar las decisiones tomadas. Esto nos llevó a elaborar un documento que pudiera ser sometido a análisis crítico, en términos de Stenhouse (1987) que presentara las hipótesis curriculares que como equipo técnico estábamos priorizando. Estas fueron definidas siguiendo las normativas promulgadas por el Consejo Federal de Educación en el marco de las políticas de inclusión educativa impulsadas en la región: Prioridad del sujeto adulto, Organización del curriculum por módulos, Campos de contenidos en diálogo con capacidades, Foco en situaciones problemáticas, Trabajo por proyectos.

El documento elaborado se presentó en un primer encuentro con inspectores en jefe ${ }^{4}$ en el Consejo Provincial de Educación, haciendo referencia además a todo el trabajo de indagación documental realizado. La intención fue que los inspectores, grandes conocedores del territorio real, pudieran advertir riesgos, y sugerir aportes para poder comenzar la tarea. Centralmente, además, esto evidenciaba el lugar de trabajo colaborativo que queríamos generar.

Una vez realizada esta primer instancia de deliberación, se organizaron reuniones con los inspectores de cada Región ${ }^{5}$. En las reuniones se agrupaban regiones cercanas y se prolongaban durante de todo el día de modo de promover debates en profundidad sobre los distintos aspectos a tratar. En ellas, se presentaba la versión preliminar de las definiciones preliminares del diseño. Luego, reunidos en pequeños grupos de inspectores, realizaban sugerencias y correcciones respecto de lo que el equipo de especialistas curriculares habían comenzado a elaborar. Este fue un momento de absoluta relevancia: las voces de los actores se sumaban a la producción. Entre los aportes mencionamos: el pedido de inclusión de educación sexual integral, la validación de las situaciones problemáticas seleccionadas en la primera versión de la propuesta curricular, y del análisis de los contenidos presentados se 
sugirieron reformulaciones en Ciencias Sociales y Naturales. Nuevamente, las voces de los actores territoriales eran parte de la definición curricular.

En función de estos aportes, el equipo de especialistas curriculares de la Dirección de Adultos reformuló el documento preliminar, y con una segunda versión de la propuesta se realizaron consultas a partir de una ronda de visitas a las escuelas, con el propósito de amplificar el proceso deliberativo incluyendo a diversos actores -en especial, a los maestros, además de analizar su viabilidad. Cabe señalar, también, los aportes de las áreas de Educación Artística y Educación Física de la Secretaría de Educación provincial, a los saberes curriculares que allí se incluyen. Consideramos central este dispositivo de elaboración deliberativa y colaborativa. Las voces autorizadas en la definición curricular no fueron solamente la de los especialistas curriculares y la de los inspectores: el equipo del área recorrió la provincia dando a conocer las versiones preliminares del diseño a maestros y directores de instituciones de toda la jurisdicción, solicitando el análisis crítico. La participación fue central en este marco: se trato de consultas durante tiempos prolongados, recorriendo el territorio, dialogando con actores diversos, en la construcción de un modelo de participación que, de algún modo, replicaba la tradición de la alfabetización de adultos en la provincia. Se trataba de reinventar las recorridas, las conversaciones, el compromiso y la mística propia de la educación de adultos.

Finalmente, se consolidó una versión final del diseño curricular para la Educación primaria de Jóvenes y Adultos cuyos rasgos centrales se presentarán en el parágrafo 3 de este trabajo.

\section{Aportes de la política curricular en pos de la inclusión educativa}

Antes de avanzar en la presentación de los rasgos centrales del diseño curricular para la escuela primaria de adultos, nos resulta indispensable comentar cuáles han sido los aportes de la literatura que analiza las políticas curriculares impulsadas por la región a partir de los años 90, con el fin de dar respuesta al desafío de la inclusión educativa. Distintos países de Latinoamérica se involucraron en importantes procesos de reformas educativas que incluyeron variadas modificaciones en los sistemas educativos de la región: en la estructura de los niveles educativos, la extensión de la obligatoriedad escolar, y procesos de descentralización que afectaron el financiamiento, la administración y la gestión de los sistemas. En el centro de las citadas reformas, la inclusión educativa ha sido un motivo principal de esas políticas. El propósito de la inclusión configuró un conjunto de exigencias, asociadas a un generalizado aumento de la escolaridad y de la obligatoriedad escolar (Tedesco y López; 2002; Tenti, 2009). De allí la importancia que adquirieron distintos programas que procuraron atender los problemas de reducción del fracaso, mantenimiento de la escolaridad y mejora de los aprendizajes de alumnos de sectores desfavorecidos (Feldman, Atorresi, Mekler, 2013; Feldman, Amantea, Feced, 2014). 
En el marco de estos intensos movimientos de reforma se avanzó en la definición de pautas curriculares para distintas modalidades educativas; entre ellas, las modalidades de Educación Técnico Profesional y la Modalidad de Jóvenes y Adultos ${ }^{6}$.

La literatura que analiza las reformas curriculares de los últimos 40 años en clave inclusión educativa, señala un conjunto de principios/rasgos generales/criterios curriculares que se constituyen en tendencias actualmente vigentes (Cox, 2004 y 2006; Dussel, 2006; Coll y Martín, 2006; Meyer, 2006; Gajardo, 1999; Kaufman y Nelson, 2005; Kohan, 1996; Krawczyk y Vieira, 2007). De esos principios, destacamos aquellos que han tenido mayor impacto en la definición del curriculum de la Modalidad de Educación de Adultos, referidos a a) avanzar hacia la integración del curriclum, b) la contextualización del contenido y c) la flexibilización curricular.

a) Desde la sociología del curriculum, Bernstein $(1995,1998)$ primero y algunos seguidores después, han planteado los límites de los curricula de alta clasificación que obturan el diálogo entre disciplinas y formatean una dinámica de trabajo en las escuelas en las que cada profesor es amo y señor de su materia. Lo anterior deja librada la relación pedagógica (lo que el autor denomina "enmarcamiento") a la potestad del docente por sobre cualquier otra autoridad y la primacía de la lógica disciplinar para el tratamiento de ciertos problemas de la vida por fuera de la escuela. La clasificación por materias aisladas impone a la vida escolar una dinámica en la que el diálogo del estudiante con el conocimiento es mediado por la disciplina y sus problemas "internos", sin reconocer que el mundo social y natural propone problemas complejos e interdisciplinarios.

Los movimientos de integración del curriculum abonan por hacer lugar a distintas instancias de diálogo entre asignaturas que "obliguen” a los profesores a trabajar en conjunto algunos temas y/o problemas que ameritan miradas compartidas e interdisciplinarias (Bourdieu y Gross, 1990): “La aspiración a una enseñanza más coherente debe favorecer las enseñanzas impartidas en común por profesores de distintas especialidades, e incluso un replanteamiento de la actual división del saber en 'disciplinas’” (p. 419). La integración curricular no tira por la borda los aportes fundamentales de las lógicas disciplinares, sino que podría combinarlos con momentos de diálogo y trabajo compartido entre más de una de ellas.

La historia del currículum muestra cómo los intentos de estructuración del contenido curricular a partir de modelos interdisciplinarios han sido seguidos por iniciativas de reestratificación del currículum en torno a las disciplinas tradicionales (Goodson, 1995 y 2000). Y la conservación de las disciplinas como base para la organización del currículum ha constituido, según Goodson, su mejor protección contra iniciativas de reformas integrales, pues permiten mantener las innovaciones dentro del microcosmos de cada asignatura; más aún, fraccionan cualquier intento de revisión de los propósitos políticos y sociales de la educación, y limitan la esfera de acción de los agentes de cambio.

b) Avanzar hacia el predominio de códigos que se orientan al conocimiento contextual y socialmente situado (Coll y Martin, 2006; Perrenoud, 2003). Por el contrario, históricamente el curriculum ha favorecido el predominio de códigos que privilegian el 
conocimiento abstracto, académico y descontextualizado tiene su justificación por su ligazón con ámbitos académicos y su asociación con exámenes externos, que las convierten en un conocimiento abstracto, formal y académico. La sobrecarga de contenidos que caracteriza a los curricula de la educación obligatoria en muchos países es, en realidad, el resultado de la aplicación reiterada de una lógica esencialmente acumulativa en los sucesivos procesos de revisión y actualización del currículo escolar en espejo con la producción de conocimiento en ámbitos académicos.

No obstante ello, a la definición abstracta y académica del contenido se viene imponiendo otro modo de abordar la identificación y definición de qué hay que enseñar y aprender en la educación en términos de competencias ${ }^{7}$ (Coll y Martín, 2006; Perrenoud, 2003). En la medida en que el concepto de competencia remite a la movilización y aplicación de saberes y tiene siempre, en consecuencia, un referente o un correlato en el comportamiento, la entrada por competencias en el establecimiento del currículo ayuda efectivamente a definir los aprendizajes básicos, imprescindibles y deseables. En este punto, un aporte importante de los estudios del curriculum nos muestran una tendencia a repensar el currículo escolar desde lo esencial, lo imprescindible, lo irrenunciable, y descargarlo del exceso de contenidos que lo caracteriza actualmente en la mayoría de los sistemas educativos.

c) Hacia la flexibilización curricular entendida como la posibilidad de otorgar mayor autonomía en las decisiones sobre el recorrido formativo a los propios estudiantes (Baquero et al, 2009; Dussel, 2008; Feldman, 2009; Jacinto y Terigi, 2007). La histórica rigidez en los trayectos formativos y en los regímenes de calificación y promoción no tiene en cuenta los diversos puntos de partida de los estudiantes del nivel, y en consecuencia concentra una enorme dispersión en términos de posibilidades de éxito. El curriculum puede mejorar y hacerse más flexible (menos cursos a la vez, más optatividad, mayor integración de materias, cuatrimestralización en el caso que se considere pertinente) y puede hacerse más atractivo (mejorando la definición del contenido por la vía del tratamiento de problemas significativos y contextualizados). Es en el régimen académico donde la flexibilización del curriculum se materializa y mejor se establecen las diferencias que permiten considerar la atención a las trayectorias diversas de los estudiantes.

\section{El caso del diseño curricular de la escuela primaria para la Modalidad de Educación de Jóvenes y Adultos: los rasgos centrales}

Este apartado tiene un doble propósito: por un lado, mostrar cómo la preocupación por la inclusión educativa se conjuga con la reconceptualización del sujeto de la educación de adultos, como aspecto central a partir del cual se revisa la formación para la modalidad y se toman decisiones sobre el curriculum; por otro lado, mostrar cómo los principios curriculares analizados en el apartado anterior se pusieron en juego como criterios para la elaboración del caso en cuestión. 
Acerca de la educación de jóvenes y adultos en la provincia de Buenos Aires: una reconceptualización necesaria

La Educación Formal de Adultos en la provincia Buenos Aires se inicia con la creación de la Dirección General de Escuelas durante la gestión de Domingo Faustino Sarmiento en 1875. Funcionaba en los terceros turnos (turno vespertino) de las escuelas primarias comunes, intentando garantizar la terminalidad de la formación del sujeto adulto. Recién en 1973, se crea la "Dirección de Educación de Adultos", dependiente de la Subsecretaría de Educación ${ }^{8}$. Esta decisión de política educativa generó condiciones para la autonomía y el funcionamiento de Escuelas y Centros de Educación de Adultos (C.E.A.) con independencia de las Escuelas Primarias para niños y la cobertura de cargos directivos y cuerpo de inspectores propios.

Sin embargo, en el marco de los procesos políticos en Argentina, durante la última dictadura militar, estas instituciones fueron afectadas con desapariciones de personas, destierros y cesantías de maestros, alumnos, referentes institucionales y comunitarios; cierre de servicios educativos, anulación de propuestas metodológicas en el campo pedagógicodidáctico específicas para esta educación dado que estaban basadas en los postulados de la pedagogía emancipadora; prohibición del desarrollo de clases a partir de propuestas grupales de trabajo; instalación impuesta del aprendizaje basado en la enseñanza individualizada; desamparo gremial, congelamiento político-cultural de todas las potencialidades articuladoras y hacedoras de experiencias de cambio social; exclusión de sectores sociales de los beneficios educativos a partir de la no apertura de escuelas y centros aún cuando la necesidad estaba presente; prohibición de publicaciones de textos académicos y líneas de investigación; establecimiento de planteles de conducción de la Educación de Adultos (Inspectores de Área Curriculares y Jefes de Región) que no provenían de esta modalidad y cuyo único propósito era controlar, cesar e invisibilizar a los actores y escenarios donde se llevaban a cabo los procesos educativos.

Con el retorno a la vida democrática en 1983, se inicia un proceso de reconstrucción de la Educación de Adultos. En este proceso de construcción socio-histórica se fue construyendo una escolaridad con características flexibles y con normativas que posibilitaron sucesivas rupturas con los determinantes duros de la escuela tradicional para esta modalidad. Pero es recientemente que la provincia de Buenos Aires en el marco de la Ley de Educación Nacional No 26.206 (2006), la Ley Provincial de Educación N 13.688 (2007) y un conjunto de normativa sancionada por el Consejo Federal de Educación citadas más arriba, buscan fortalecer la identidad de la modalidad de educación de adultos.

En las últimas décadas, se han producido cambios en el conjunto social que afectan tanto la composición como las demandas en torno de la educación de adultos, sin que se hayan producido modificaciones estructurales en sus aspectos organizativos y curriculares. Es en este sentido, que la educación de adultos, nombrada en la actualidad como educación de jóvenes y adultos, incorpora en sus instituciones cada vez un número mayor de jóvenes, adolescentes y adultos que traen consigo un conjunto de prácticas sociales propias y que interpelan a las instituciones otrora pobladas casi exclusivamente por adultos. ${ }^{9}$ 
Por ello, era preciso que la escuela de jóvenes y adultos sea promotora de espacios sociales y centro de la vida cultural de la comunidad dentro de la cual está inmersa. Al mismo tiempo, es necesario profundizar articulaciones en dos sentidos: por un lado, entre la propuesta educativa y los saberes ligados al mundo del trabajo, no sólo con criterios de inserción en la estructura ocupacional sino como saberes más generales vinculados al ámbito de la ciudadanía, en tanto los sujetos que la transitan y aquellos a quienes aún se debe incorporar, son portadores de derechos sociales; por otro lado, entre esta propuesta y la continuidad con otras ofertas educativas que se abren después de obtener la finalización de la primaria, en especial, con los estudios secundarios.

Esto marca al mismo tiempo un profundo cambio y una continuidad en la concepción política-pedagógica de los sujetos destinatarios -siempre diversos, múltiples, portadores de prácticas, saberes, valores y hábitos diferentes entre sí- y se traduce en una nueva estructura para la educación primaria de jóvenes y adultos, que garantice la inclusión, la permanencia y la acreditación del nivel para todas las personas mayores de 14 años de la provincia de Buenos Aires. Para el logro de dichos propósitos, se impone la necesidad de una propuesta de enseñanza, acorde con los lineamientos de política educativa vigentes, que sea capaz de incorporar también a todos quienes hoy no están en sus aulas.

La "Educación de Adultos" es una construcción socio-histórica compleja, asociada íntimamente al proceso de formulación de demandas por educación de jóvenes y adultos de los sectores populares. Esto pone en evidencia la existencia de distintos mecanismos de exclusión escolar que afectan a determinados sectores sociales desfavorecidos. Entendemos que la marginación y el fracaso escolar se producen en el marco de procesos de desigualdad más amplios vinculados a la vulneración de otros derechos humanos, como la vivienda, el trabajo, la salud, la participación social, entre otros. Quienes concurren a las escuelas de adultos ya han pasado por experiencias de escolaridad que los marcaron, incidiendo en la percepción que poseen de sí mismos. En este sentido, existen huellas que condicionan la posibilidad de que los sujetos se reconozcan así mismos como personas capaces de aprender, muestran falta de confianza y desvalorización de sus propios conocimientos. Para los adultos, finalizar su escolaridad primaria representa mucho más que la obtención de un título, los habilita para percibirse capaces de continuar aprendiendo y de valorar sus capacidades.

Los principios que guiaron la elaboración del diseño curricular en el debate con los actores del sistema educativo provincial

Los principios generales que guiaron las primeras versiones de la propuesta curricular que se puso a consideración de los distintos actores de la modalidad (en especial, inspectores y docentes), recuperan los conceptos de integración curricular, contextualización del contenido y flexibilización del curriculum; y son los siguientes:

1. La autonomía de los ciclos formativos: los ciclos formativos no son anuales, como es tradición en el sistema educativo. El análisis de los recorridos previos de los estudiantes, sus biografías, los saberes alcanzados, se proponen para ser considerados 
en las decisiones del recorrido formativo del estudiante. No se trata de un único recorrido para todos en sintonía con la tendencia hacia la flexibilización curricular.

2. La opción por una secuencia no lineal para organizar los módulos que componen el diseño curricular: las decisiones acerca de este tipo de organización curricular suponen un trabajo de enseñanza complejo, espiralado y diverso. Esta enunciación se concreta en la definición de situaciones problemáticas a ser abordadas en diálogo con la tendencia de integración curricular.

3. El diálogo con el contexto local: se propone que las propuestas de enseñanza se vinculen con el territorio, a partir de las potencialidades y límites que son propios de cada institución educativa y de sus ámbitos de influencia, recuperando la preocupación por la contextualización del contenido del curriculum.

Se proponen tres ciclos formativos para organizar el Nivel Primario: el Ciclo de alfabetización, que tiene como propósito, en el marco de los procesos de alfabetización, favorecer la construcción de autonomía y generar estrategias de participación ciudadana; el Ciclo de Formación integral que se centra en el desarrollo de capacidades vinculadas con el conocimiento de la lengua-escrita y oral, la matemática, las ciencias y tecnologías de la información y comunicación, las artes, la formación ética, la formación para el mundo del trabajo y el cuidado ecológico; y el Ciclo de Formación por Proyectos, que se propone generar un espacio pedagógico que permita resignificar los aprendizajes de las áreas curriculares en el análisis y resolución de nuevos problemas de relevancia social para los estudiantes, su contexto socio - cultural y desarrollo local.

Asimismo, la organización de la propuesta curricular se realiza a través de módulos. Entendemos por módulo a un componente curricular referido a un campo de contenidos que constituye una unidad de sentido que organiza la enseñanza y el aprendizaje, con un importante grado de autonomía en relación con la estructura curricular de la que forma parte. La estructura modular supone asumir a la educación en directa conexión con los procesos educativos vinculados al desarrollo social, cultural, económico y productivo de las personas en comunidad, recuperando y valorando la tradición en la modalidad de adultos iniciada por Paulo Freire. Tradición que continúan en la región los trabajos de Rosa María Torres (2001) y Violeta Núñez (1999) quienes permiten pensar en una pedagogía con fuertes lazos en el territorio. En línea con Rosa María Torres, la organización modular que adopta esta propuesta curricular, invita a pensar lo educativo desde el desarrollo local, desde la idea de comunidad de aprendizaje definida por la autora como una comunidad humana organizada que construye y se involucra en un proyecto. En esta propuesta curricular el módulo organiza las capacidades en las que se pretende formar y articula los materiales educativos, los momentos pedagógicos, la intencionalidad educativa y los procesos evaluativos. En este marco, permite integrar diferentes áreas disciplinares. En la gestión de la clase propiamente dicha, implica un espacio pedagógico dinamizador de los procesos de construcción colectiva de conocimientos, dando forma a las citadas “comunidades de aprendizaje” (Rosa María Torres, 2001).

La definición de la propuesta modular no es una definición meramente metodológica. Implica considerar la especificidad del sujeto adulto, como partícipe y constructor de la 
cultura. Impacta y define el enfoque de enseñanza, y propone una perspectiva diferente a la centralidad que el contenido tiene en la modalidad de educación primaria común. La estructura modular se sostiene en base a criterios de flexibilidad en tiempo y espacio y de apertura hacia la realidad de cada estudiante, contextualizando los contenidos de enseñanza. Los módulos permiten una multiplicidad de formas de articularlos entre sí, construyendo la formación de cada estudiante con una trayectoria que no pretende ser homogénea. Los módulos abordan diferentes situaciones problemáticas que toman forma pedagógica a partir de la elaboración de proyectos en los que se proponen actividades que responden a necesidades sociales significativas del contexto local, provincial o nacional. Ambos componentes son una forma de apropiarse, construir y organizar el conocimiento promoviendo aprendizajes significativos y productivos para las personas jóvenes y adultas, por lo que se los consideran generadores de procesos de aprendizaje. Las situaciones problemáticas y los proyectos, a nivel jurisdiccional, se identifican a partir de Contextos Problematizadores que fueron definidos a nivel nacional.

Figura 1: Ejemplo de una de las propuestas del Diseño

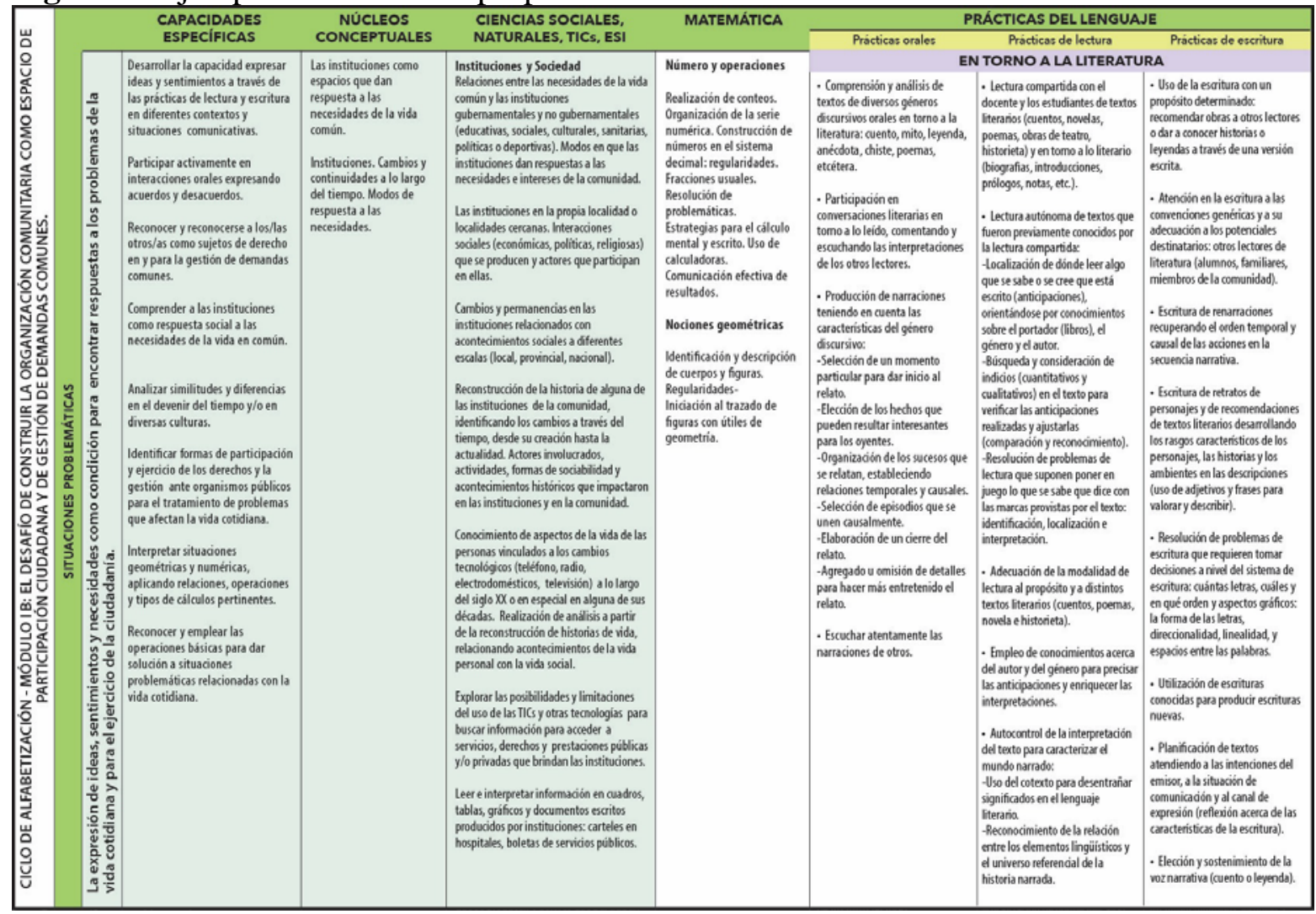

Fuente: Diseño Curricular para Educación Primaria de Jóvenes y Adultos (2017) 
En relación con la enseñanza, la definición de contextos problematizadores y situaciones problemáticas intentan organizar las decisiones acerca de cómo enseñar. Para ello, en los “cuadros" elaborados para cada módulo, la lectura horizontal permite articular cada situación problemática con un conjunto de núcleos conceptuales, que abren la situación problemática en dimensiones del contenido a considerar para trabajar con los adultos. Cabe destacar que las situaciones problemáticas se definen a partir de las ciencias sociales y naturales, con énfasis en problemas significativos. En este sentido, la identificación de núcleos conceptuales de cada área o campo de conocimiento, en diálogo con las capacidades específicas, requieren ser consideradas como las orientaciones principales para poder definir la enseñanza concreta en las aulas.

Específicamente, en términos de estrategias privilegiadas para la enseñanza, se considera la resolución de problemas porque promueve la búsqueda de respuestas a distintas situaciones a través de diversos caminos y el análisis de la adecuación y factibilidad de esa respuesta. Es tarea del docente, en el momento de realizar su planificación y en conocimiento del grupo de estudiantes y la realidad local, contextualizar la propuesta curricular y situar la enseñanza en el marco de los problemas más significativos para cada comunidad. En este sentido, los módulos orientan la posibilidad, pero la definición del alcance de los contenidos de las distintas áreas requieren de la decisión del docente. Cabe señalar que en esta definición resulta relevante el diálogo con los estudiantes. Consideramos relevante que los problemas que se definan tengan el grado de dificultad necesario para ser un desafío intelectual, pero no estar tan alejado de las posibilidades de resolución de los estudiantes, que no les permita acceder a los contenidos necesarios para su resolución.

En relación con la centralidad del trabajo por proyectos, se lo propone como estrategia de planificación de la enseñanza y como modalidad organizativa privilegiada para llevar adelante el trabajo. Definir en el aula el trabajo por proyectos implica asumir una perspectiva situada en relación con la enseñanza (Díaz Barriga, 2005). Llevar adelante un proyecto, constituye un desafío en el que participan colaborativamente estudiantes, docentes, y la comunidad educativa en general. Se propone el trabajo colaborativo y la construcción del conocimiento, en la búsqueda de una solución. La configuración de la enseñanza en el marco de la formulación de proyectos, intenta destacar la multidimensionalidad de la realidad: la relación con el entorno social, político, económico, cultural, ecológico e institucional. Esta configuración promueve que los sujetos piensen críticamente la realidad en que están inmersos para tomar conciencia de su importancia y poder generar acciones transformadoras.

En este marco, es imprescindible poder identificar las necesidades y los intereses de cada grupo de alumnos, conocer sus diferencias individuales y sus características grupales dado que esta contextualización es central para la enseñanza. Se trata de alumnos con estilos de aprendizaje, niveles de conocimientos, ritmos, actitudes, trayectorias y experiencias e intereses diferentes. El hecho de reconocer esas diferencias trae implicancias en las decisiones de planificación y en la puesta en marcha de la enseñanza. La consideración y el respeto por la diversidad y heterogeneidad de los grupos de estudiantes, que es ineludible a la hora de considerar la enseñanza. 
El desafío entonces puede definirse a partir de la pregunta ¿cómo pensar propuestas de enseñanza para aulas heterogéneas? Según Anijovich y otros (2004), considerar el aula heterogénea implica ubicarse desde el paradigma de la diversidad. Atender a la diversidad supone tomar decisiones políticas y pedagógicas, implica debatir y lograr consensos acerca de qué es lo común que todos los estudiantes deben aprender y utilizar estrategias diferentes para que todos lo logren. Una pedagogía de la diversidad no puede apoyarse en la homogeneidad de formas de trabajar.

En cuanto a las orientaciones para la evaluación y la acreditación de los módulos se propone un enfoque de la evaluación participativa, formativa e integradora; que prevea instancias de evaluación grupal e individual. En el aula, requiere considerarse vinculada a la continuidad y no solamente a la finalización de un proceso. De acuerdo con (Anijovich y Cappelletti, 2017), la evaluación formativa ocurre en paralelo al proceso de enseñanza y de aprendizaje e implica recoger información que permita al docente, comprender los conflictos que surgen en el desarrollo de la propuesta de enseñanza y tomar las medidas necesarias para reajustar lo planificado; y al estudiante, conocer qué debe modificar para seguir avanzando en el proceso de aprendizaje. Supone, instancias de devolución a los estudiantes acerca (y durante) el recorrido transitado. La evaluación implica un conjunto de acciones continuas y sostenidas en el desarrollo del proceso que permitan obtener información y dar cuenta de cómo se desarrollan los aprendizaje de los alumnos tanto como los procesos de enseñanza en relación con la posibilidad de ajustar, en la propia práctica, los errores o aciertos de los procesos de enseñanza propuestos-. Al evaluar, se busca información de muy diversa índole; a veces, conocer las ideas que los estudiantes traen construidas con anterioridad, en otras ocasiones, conocer la marcha de un proyecto, en otras, el aprendizaje de ciertos procedimientos; finalmente el aprendizaje como rendimiento o logro al final de un proceso formativo.

En la educación de jóvenes y adultos resulta conveniente la valoración cualitativa más que la cuantitativa. Una devolución cualitativa resulta de mayor utilidad tanto para el docente como para el estudiante, dado que se vincula realmente con los logros y con las dificultades con las que todavía deben trabajar. Al estudiante, le permite poner en juego estrategias para mejorar sus aprendizajes. Pero también, colabora en los procesos reflexivos de los docentes, y los ubica en situaciones donde es posible que revisen las propias situaciones de enseñanza para tomar decisiones de cómo intervenir.

Por lo anterior, una práctica deseable podría recurrir al uso de variados instrumentos de evaluación dado que se hace indispensable para obtener información sobre distintos aspectos del proceso de aprendizaje.

El contexto de la evaluación puede promover en los estudiantes una creciente autonomía en la toma de decisiones y en la regulación de sus aprendizajes, favoreciendo el pasaje desde un lugar de heteronomía -donde es el docente quien propone las actividades, los eventuales caminos de resolución y las evaluaciones, y el alumno es quien las realiza- hacia un lugar de mayor autonomía en el que el estudiante pueda plantearse problemas, seleccionar sus propias estrategias de resolución, planificar el curso de sus acciones, administrar su tiempo y realizar evaluaciones parciales de sus propios procesos, reconociendo logros y dificultades. 
En los debates con inspectores y docentes se planteó considerar otros aspectos ligados a la evaluación y que se vinculan con los desempeños más globales de los estudiantes frente al conocimiento: el trabajo de construcción de conocimiento, en la medida en que todos participan individual y grupalmente de la construcción de modelos explicativos, del diseño e implementación de proyectos, y de las actividades generales de aprendizaje que se propongan. Por lo tanto, se propone que la evaluación incluya este aspecto social, dando oportunidades a los estudiantes para realizar también evaluaciones del propio desempeño tanto como el de sus compañeros. Esta responsabilidad de evaluar desempeños, implico, asimismo, considerar un segundo aspecto, vinculado con la democratización de las relaciones en el aula y el aprendizaje, para los cuales una evaluación deberá estar fundamentada en criterios explícitos y no en cuestiones de índole personal -simpatía o antipatía por un compañero o un argumento-. De modo que es fundamental que el estudiante aprenda a evaluar la marcha de un proyecto o el desempeño dentro de un grupo, estableciendo conjuntamente y con la ayuda del docente cuáles serán los criterios con que es conveniente juzgar la pertinencia de cierto argumento o el cumplimiento de las normas para el trabajo. Por último, la posibilidad de reflexionar sobre la marcha de los aprendizajes, a partir de criterios que fueran explicitados y compartidos, ayuda a repensar los aspectos que no han quedado lo suficientemente claros, así como a plantear caminos de solución.

Se propusieron como alternativas a la estrategia clásica de la evaluación centrada en la figura del docente como único evaluador, las siguientes:

La evaluación entre pares o evaluación mutua, en donde el estudiante comparte con sus pares los criterios de evaluación construidos con el docente, y en función de ellos, puede hacer señalamientos sobre los aspectos positivos o a mejorar, tanto del desempeño individual como el grupal en relación con la tarea establecida. Este tipo de evaluación, por supuesto debe ser supervisada por el docente, puede aportar información acerca de la capacidad de los estudiantes para argumentar y sostener criterios frente a otros. La coevaluación, entendida como una guía que el docente brinda a sus estudiantes durante la realización de una tarea, indicando no sólo la corrección o incorrección de lo realizado, sino proponiendo preguntas o comentarios que orienten a los alumnos hacia el control de sus aprendizajes, llevándolos a contrastar los objetivos de la actividad con los resultados obtenidos y tendiendo siempre hacia la autorregulación.

La autoevaluación del estudiante supone la necesidad de contar con abundante información respecto a la valoración que es capaz de hacer de sí mismo y de las tareas que realiza. La autoevaluación no consiste en hacer que el alumno corrija su prueba escrita siguiendo los criterios aportados por el docente, sino más bien, en un proceso en el cual el estudiante pueda gradualmente lograr la anticipación y planificación de sus acciones y la apropiación de los criterios de evaluación. 


\section{Los pendientes}

Toda propuesta curricular, en tanto se construye históricamente sobre una propuesta anterior, implica cierto monto de innovación. En ese sentido, entendemos el curriculum en términos de un punto de partida que recupera prácticas preexistentes y abre un horizonte a alcanzar. ¿Cuán cercana (o lejana) es la propuesta curricular de las posibilidades de implementación? Este problema es central en el marco de los procesos de definición curricular, y puede abordarse considerando diferentes perspectivas. En tanto las políticas educativas crean condiciones (o no) para llevar adelante los procesos educativos, la elaboración de una propuesta curricular podría acompañarse con apoyos para su implementación. En el escenario actual, la propuesta curricular elaborada para el nivel primario de la educación de jóvenes y adultos exige ciertas condiciones en la práctica pedagógica: propuestas globalizadas de enseñanza, recorridos de enseñanza diversos, evaluaciones que se alejan de las modalidades habituales, entre otras. Estas prácticas se alejan de las prácticas tradicionales y exigen posicionamientos docentes, modalidades de planificación, diseños de actividades y tratamientos de los saberes y propuestas de evaluación que no necesariamente han sido abordados en los procesos de formación docente inicial. Un modo de acompañar la implementación del diseño curricular es la formación docente en servicio: destinar jornadas al trabajo con los docentes en las escuelas primarias será una condición clave para el desarrollo de la propuesta. Además, la elaboración de materiales curriculares que pongan en juego y modelicen la enseñanza y la evaluación puede resultar en recursos potentes y en puntos de partida para acompañar a los docentes. Ambas líneas de trabajo se han iniciado, sólo resta estar atentos a lo vaivenes de la política educativa...

\section{Notas}

1 Finalmente, el Diseño Curricular para la escuela primaria de Adultos fue aprobado en la Ciudad de La Plata, el 13 de julio de 2017. RESFC-2017-45-GDEBA-DGCYE.

2 Ministerio de Educación de la Nación, Resolución del CFCyE Nº 225/04, del 11 de agosto del año 2004.

3 Algo similar sucederá en el caso que estamos presentando a partir de una concepción diferente del Sujeto Adulto, a la sostenida históricamente que está en el centro de la propuesta del curriculum de la modalidad.

4 Concurrieron a la reunión aproximadamente 30 inspectores, representando el universo total de los mismos. Cabe señalar que no existía tradición de convocarlos desde las autoridades políticas de la provincia, mucho menos para una reunión de presentación de hipótesis de propuesta curricular.

5 La provincia de Buenos Aires se subdivide territorialmente en 25 regiones. Existen inspectores en jefe regionales y distritales del área de Adultos. No es posible establecer una correspondencia numérica entre regiones e inspectores, dado que están atravesadas por variables diversas como por ejemplo, la distribución territorial de la oferta. Los sujetos que asumen cargos de inspección en el área, acceden por concurso y casi en su totalidad han tenido trayectoria previa en el área de Adultos. Son conocedores del campo de la práctica de la educación de Jóvenes y Adultos.

6 La elaboración del marco normativo de estas reformas se hacen posibles a partir de la Ley Nacional de Educación $\mathrm{N}^{\mathrm{o}}$ 26.206 del 2006. Para la Modalidad de Educación de Jóvenes y Adultos ver ARGENTINA, Resolución CFE N 118/10, "Lineamientos curriculares para la educación Permanente de Jóvenes y adultos; Hacia la Estructura Curricular de la Educación Permanente de Jóvenes y Adultos” y Resolución CFE N 254/15, “Marcos de Referencia para la Modalidad de Educación Permanente de Jóvenes y Adultos- Lineamientos para la construcción de diseños y/o planes de estudio 
jurisdiccionales”. Para la Modalidad de Educación Técnico Profesional, ver ARGENTINA, Ley de Educación Técnico Profesional No 26.048, del año 2005. Resolución CFCyE No 261/06 ANEXO: "Proceso de homologación y marcos de referencia de títulos y certificaciones de Educación Técnico Profesional”. Resolución CFE No 47/08 ANEXO I: "Lineamientos y criterios para la organización institucional y curricular de la Educación Técnico Profesional correspondiente a la Educación Secundaria y la Educación Superior”.

7 Usamos el concepto de competencias desde la perspectiva planteada por Philippe Perrenoud (2003).

En el Documento Hacia la Estructura Curricular de la Educación Permanente de Jóvenes y Adultos (2011) se presentan doce Contextos Problematizadores que se consideraron de mayor incidencia en el desarrollo individual y colectivo de los/las estudiantes de la EPJA

1. Dicotomía entre el desarrollo y el cuidado de la naturaleza.

2. Naturalización de la inequidad en el acceso a las determinantes de la salud.

3. El mundo del trabajo en la nueva configuración social, política y cultural.

4. Las relaciones asimétricas de género y su incidencia en la construcción de identidades.

5. Diversidad sociocultural y desigualdad.

6. El Sujeto ante un nuevo contexto histórico: de la ciudadanía formal a las prácticas emancipadoras.

7. El poder de lo comunicacional y su incidencia en la construcción de identidades autónomas individuales y colectivas.

8. La apropiación cultural y las tensiones interculturales.

9. Desnaturalización de lo tecnológico: visibilizar la producción científica en lo cotidiano.

10. El desafío de construir la organización comunitaria como espacio de participación ciudadana y de gestión de demandas comunes.

11. Derecho a la tierra y vivienda: Identidad, dignidad y oportunidades para todos.

12. La dimensión económica y sus efectos en la vida social.

\section{Bibliografía}

AMANTEA, A.; CAPPELlETTI, G.; COLS, E. Y FEENEY, S. "Propostas curriculares na Argentina: as tradiçoes disciplinar, da didática peral e das didáticas especiais”. En Casimiro Lopes, A. y Macedo, E. (Org.): Políticas de currículo em múltiplos contextos. Sao Paulo, Cortez Editora, 2006, Pp. 38 a 69.

ANIJOVICH, R., MALBERGIER, M., \& SIGAL, C. Una introducción a la enseñanza para la diversidad. FCE de Argentina, Buenos Aires, 2004.

ANIJOVICH, R. Y CAPPELLETTI, G. La evaluación como oportunidad. Buenos Aires, Paidos, 2017.

ARROYO, M. Educación en tiempos de exclusión. En GENTILI, P. Y FRIGOTTO, G. (comp.) La ciudadanía negada: políticas de exclusión en la educación y el trabajo. Buenos Aires, CLACSO. Colección Grupo de Trabajo BARCO, S (Coord.), 2001.

BARCO, S. "Del orden, poderes y desorden”. ICKOWICZ, M; IURI, T.; TRINCHERI, A. Universidad, docentes y prácticas. El caso de la UNCo. EDUCO, UNCo, 2005. Cap.II, pág 47 a 72.

BAQUERO, R., TERIGI, F., TOSCANO, A.G., BRISCIOL, B. Y SBURLATTI, S. "Variaciones del Régimen Académico en Escuelas Medias con Población Vulnerable. Un Estudio de Casos en el Área Metropolitana de Buenos Aires"; REICE. Revista Iberoamericana sobre Calidad, Eficacia y Cambio en Educación, 7(4), 2009, en: http://www.rinace.net/reice/numeros/arts/vol7num4/art15.pdf

BERNSTEIN, B. “Clasificación y enmarcación del conocimiento educativo”. En Revista Colombiana de Educación. 1er. Semestre, 1985.

BERNSTEIN, B. Clases, códigos y control. Madrid, Akal, 1988. 
BOURDIEU, P. Y GROSS, F. “Principios para una reflexión sobre los contenidos de la enseñanza”. En Revista de Educación. Madrid, MEC, 1990, No 292. Pp 417-425.

BROOKHART How to create and us rubrics for formative assessment and grading. Alexandria, VA: association for Supervision and curriculum development, 2013.

COLL, C. Y MARTIN, E. Vigencia del debate curricular. Aprendizajes básicos, competencias y estándares. OREALC/UNESCO. Santiago de Chile, 2006.

COX, C. "Reformas curriculares y evaluación internacional de resultados de aprendizaje categorías analíticas y datos para la reflexión”. Material de apoyo para actividades del Seminario de discusión de la problemática educativa actual para cuadros superiores de Ministerios de Educación de las Provincias, organizado por Ministerio de Educación, Ciencia y Tecnología de Argentina y el IIPE,2004.

COX, C. "Construcción política de reformas curriculares: el caso de chile en los noventa" Revista de currículum y formación del profesorado, 10, 1, 2006.

DUSSEL, I. "Estudio sobre gestión y desarrollo curricular en países de América Latina”. Ponencia presentada en el contexto de la Segunda Reunión del PRELAC, OREALC/UNESCO. Santiago de Chile, 2006. http://www.elcorreo.eu.org/IMG/pdf/doc-1252.pdf

DÍAZ BARRIGA, F. Enseñanza situada. Vínculo entre la escuela y la vida. México: McGraw Hill, 2005.

DUSSEL, I. La escuela media y la producción de la desigualdad. En: Tiramonti, G. y Montes, N. (comp.) “La escuela media en debate. Problemas actuales y perspectivas desde la investigación. Buenos Aires, FLACSO- Ediciones Manantial, 2008.

FEENEY, S. “La Política curricular en Argentina: ¿todo vale?”. En Montero, A. (Org.): Novas subjetividades, currículo, docência e questoes pedagógicas na perspectiva da inclusao social. Recife, Ediçoes Bagaço, 2006, Pp. 341 a 354.

FEENEY, S. Y FELDMAN, D. “Regulaciones nacionales sobre el curriculum”. Educación en revista. [online]. 2016, vol.32, n.2, pp.19-44. ISSN 0102-4698. http://dx.doi.org/10.1590/0102-4698153047.

FELDMAN, D. "La innovación escolar en el currículum”, En: Romero, C. (comp) Claves para mejorar la escuela secundaria (pp. 63-78), Buenos Aires. Noveduc, 2009.

FELDMAN, D; ATORRESI, A; MEKLER, V. (2013): "Planes y programas para mejorar el aprendizaje y reducir el fracaso en la Educación básica en América Latina”. En Revista Latinoamericana de Educación Comparada. Número 4. ISSN 1853-3744. Pags 12-24. www.saece.org.ar/relec/numero4.php

FELDMAN, D.; AMANTEA, A.; y FECED L. (2014): "Los componentes de la evaluación en los programas de disminución del fracaso y mejora de los aprendizajes en América Latina”. Ponencia en primer encuentro internacional de evaluación UNCPBA; Tandil. 2014

FRIGERIO, G. (comp.) Curriculum presente, ciencia ausente. Normas, teorías y críticas. Buenos Aires, FLACSO- Miño y Dávila, 1991.

GAJARDO, M. Reformas Educativas en América Latina. Balance de una Década. Documento del PREAL Documentos No 15, 1999.

GENTILI, P. "Marchas y contramarchas. El derecho a la educación y las dinámicas de exclusión incluyente en América Latina (a sesenta años de la Declaración Universal de los Derechos Humanos)", en Revista Iberoamericana de Educación- OEI, Nº 49, 2009. Disponible en: http://www.rieoei.org/rie49a01.htm

GOODSON, I. Historia del currículum. La construcción social de las disciplinas escolares. Barcelona, Pomares-Corredor, 1995.

GOODSON, I. El cambio en el curriculum. Barcelona, Octaedro, 2000.

JACINTO, C. Y F. TERIGI ¿Qué hacer ante las desigualdades en la educación secundaria? Aportes de la experiencia latinoamericana. Buenos Aires, UNESCO/Santillana, 2007. 
KAUfMAN, R. Y NELSON, J. Políticas de Reforma Educativa. Comparación entre Países. Documentos de PREAL, N 33, 2005.

KOGAN, M. "Formas de gobierno y evaluación de los sistemas educativos”. En M. Pereyra, J. García, A. Gómez y M. Beas (comps.). Globalización y descentralización de los sistemas educativos. Barcelona, Ediciones Pomares Corredor,1996.

KRAWCZYK, N. R. Y VIEIRA, V. L. Homogeneidad y heterogeneidad: un estudio comparativo sobre la reforma educativa de la década del 90 en Argentina, Brasil, Chile y México. Estudios Pedagógicos XXXIII, $N^{\circ}$ 2; 2007, pp.59-80.

LUNDGREN, U. "Formulación de la política educativa, descentralización y evaluación”. En Pereyra y otros (comp.) Globalización y descentralización de los sistemas educativos. Fundamentos para un nuevo programa de educaucón comparada. Barcelona. Pomares- Corredor, 1996, pp 395 a 414.

NÚÑEZ, V. Pedagogía social: cartas para navegar en el nuevo milenio. Buenos Aires, Santillana, 1999.

PALAMIDESSI, M. "Desarrollos curriculares para la educación básica en el Cono Sur: prioridades de política y desafíos de la práctica”, IBE Working Papers on Curriculum Issues N 5. Ginebra, Suiza, 2006.

PERRENOUD, PH. Construir competencias desde la escuela. Santiago de Chile: J. C. Sáez Editor, 2003.

SCHWAB, J. Un enfoque práctico como lenguaje para el currículum. Buenos Aires, El Ateneo. 1973, (Original en inglés, 1969).

STENHOUSE, L. La investigación como base de la enseñanza. Madrid, Morata, 1987.

STENHOUSE, L. Investigación y desarrollo del curriculum. Madrid, Morata, 1991.

TABA, H. Elaboración del Currículo. Buenos Aires, Troquel,1991. $9^{a}$ ed. (Original en inglés, 1962)

TEDESCO, J. C; LÓPEZ, N. "Desafíos a la educación secundaria en América Latina”. Revista de la Cepal No 76, 2002, pag 66-69.

TENTI, E. Abandono escolar y políticas de inclusión en la educación secundaria. IIPE-UNESCO/PNUD, Buenos Aires, 2009.

TORRES, R. M. Participación ciudadana y educación. Una mirada amplia y 20 experiencias en América Latina. Documento para la OEA (Organización de Estados Americanos), Washington, 2001.

TYLER, R. Principios básicos del currículo. Buenos Aires, Troquel, 1973 (Primera edición en inglés, 1949).

\section{Correspondência}

Graciela Cappelletti: Es magister en Didáctica por la Universidad de Buenos Aires. Se desempeña como profesora adjunta regular en la Universidad Nacional de Quilmes y es investigadora de la Universidad de Buenos Aires. Enseña además en la Escuela de Educación de la Universidad de San Andrés y es Directora de la Licenciatura y del Profesorado en Ciencias de la Educación. Fue Directora de Currícula y Enseñanza del Ministerio de Educación de la Ciudad de Buenos Aires.

E-mail: gachycappelletti@gmail.com

Sivina Feeney: Magister en Didáctica de la Universidad de Buenos Aires e Investigadora Docente de la Universidad Nacional de General Sarmiento y de la Universidad de Buenos Aires.

E-mail: silvinafeeney@gmail.com

Texto publicado em Currículo sem Fronteiras com autorização das autoras 